\title{
Germanica
}

«Wenn nämlich bereits ein Wort keine Entsprechung findet, wie soll dann ein halbes Leben in der neuen Sprache erzählt werden ? » Zur Prosa Melinda Nadj Abonjis

"If no word has an exact translation, how can a life story be told when half of it is lost translation? » On Melinda Nadj Abonjis writing.

"Lorsqu'il n'y a déjà pas d'équivalent pour un mot, comment raconter une existence réduite de moitié dans la nouvelle langue? " À propos de la prose de Melinda Nadj Abonji.

\section{René Kegelmann}

\section{(2) OpenEdition} Journals

Édition électronique

URL : http://journals.openedition.org/germanica/1975

DOI : $10.4000 /$ germanica. 1975

ISSN : 2107-0784

Éditeur

Université de Lille

Édition imprimée

Date de publication : 31 décembre 2012

Pagination : 9-20

ISBN : 9782913857308

ISSN : 0984-2632

Référence électronique

René Kegelmann, « « Wenn nämlich bereits ein Wort keine Entsprechung findet, wie soll dann ein halbes Leben in der neuen Sprache erzählt werden?»

Zur Prosa Melinda Nadj Abonjis », Germanica [Online], 51 | 2012, Online erschienen am: 11 Januar 2013, abgerufen am 06 Oktober 2020. URL : http://journals.openedition.org/germanica/1975; DOI https://doi.org/10.4000/germanica. 1975

Ce document a été généré automatiquement le 6 octobre 2020.

(c) Tous droits réservés 
« Wenn nämlich bereits ein Wort keine Entsprechung findet, wie soll dann ein halbes Leben in der neuen Sprache erzählt werden? » Zur Prosa Melinda Nadj Abonjis

"If no word has an exact translation, how can a life story be told when half of it is lost translation?" On Melinda Nadj Abonjis writing. "Lorsqu'il n'y a déjà pas d'équivalent pour un mot, comment raconter une existence réduite de moitié dans la nouvelle langue? » À propos de la prose de Melinda Nadj Abonji.

René Kegelmann

\section{Melinda Nadj Abonji im deutschsprachigen literarischen Leben}

Als Melinda Nadj Abonji im Jahre 2010 sowohl den Deutschen Buchpreis als auch den Schweizer Buchpreis gewann, hatte das in mehrfacher Hinsicht Signalwirkung. Zum ersten Mal hatte eine deutschsprachige Schweizer Autorin und eine Autorin, deren Muttersprache nicht das Deutsche ist, den seit 2005 von der Stiftung des Börsenvereins des Deutschen Buchhandels verliehenen, medial ausgesprochen beachteten und hochdotierten Deutschen Buchpreis ${ }^{1}$ für den besten Roman in deutscher Sprache zugesprochen bekommen. In den Vorjahren waren auf der Shortlist des Deutschen Buchpreises bereits AutorInnen mit anderskulturellen Wurzeln - wie Saša Stanišić (2006) und Sherko Fatah (2008) - aufgetaucht, aber der Hauptpreis war bis dato AutorInnen mit deutscher Muttersprache vorbehalten². Dass der 1968 in Serbien 
innerhalb der ungarischen Minderheit geborenen Melinda Nadj Abonji, die seit 1973 in der Schweiz lebt, im selben Jahr, nämlich 2010, auch der seit 2008 vom Verein Literaturfestival Basel in Zusammenarbeit mit dem Schweizer Buchhändler- und Verleger-Verband für ein deutschsprachiges literarisches oder essayistisches Werk von in der Schweiz lebenden Autoren vergebene Schweizer Buchpreis ${ }^{3}$ zuerkannt wurde, bestätigt die zunehmende Bedeutung der Entwicklung einer literarischen Strömung, die in Deutschland oftmals als Migrantenliteratur oder Migrationsliteratur ${ }^{4}$, in der Schweiz als die Literatur sogenannter Secondos ${ }^{5}$ bezeichnet wird. Nadj Abonji, die im Alter von fünf Jahren aus der Vojvodina (Serbien) in die Schweiz eingewandert ist, gehört der Definition nach zu dieser zweiten Generation von Einwanderern.

Der Landwechsel spielt in ihrem preisgekrönten Roman Tauben fliegen auf $f^{6}$, in dem die Existenz zwischen zwei Kulturen und Ländern sehr sensibel für die Nuancen des Alltags geschildert wird, eine wichtige Rolle. Die Integrationsbemühungen der Familie Kocsis in der Schweiz und die regelmäßige Rückkehr in die Herkunftsgesellschaft der Vojvodina wird in langen verschlungenen Satzkaskaden, Vor- und Rückblenden und assoziativen Passagen ${ }^{7}$ aus der Perspektive der zum Erzählzeitpunkt bereits erwachsenen Tochter Ildikó über einen längeren Zeitraum von etwa 20 Jahren erzählt. Dabei spielt die Sprache bzw. der Spracherwerb eine zentrale Rolle. Ausgehend von dem Blick des Kindes aus der Vojvodina auf die Schweiz über erste Versuche des Spracherwerbs in der Schweiz und damit verbundene Reflexionen über Inklusion und Exklusion richtet sich der Fokus in diesem Aufsatz auf die Grenzen der Kompabilität von deutscher und ungarischer Sprache, die zugleich die Grenzen zweier Welten bezeichnen. Schließlich soll auch auf das Verhältnis von Sprache und Gedächtnis und auf die in Tauben fliegen auf angedeutete Utopie einer sprachgrenzüberschreitenden Kommunikation eingegangen werden.

\section{Der Wechsel von der Vojvodina in die Schweiz: Sprachverlust, Spracherwerb und Sprachlosigkeit}

Im Roman von Melinda Nadj Abonji beginnt der Landwechsel der Protagonistin gleichermaßen mit Verheißungen wie Schmerzen. Während die Eltern der Erzählerin in ihrem Geburtsjahr 1968 als Migranten zunächst alleine in die Schweiz gehen, um die Situation vorzusondieren, bleiben die Erzählerin Ildikó und ihre Schwester Nomi noch einige Jahre bei der Großmutter in der Kleinstadt Senta in der Vojvodina (Serbien). Dabei gilt es zu berücksichtigen, dass die Familie Kocsis in der Vojvodina zur ungarischen Minderheit, die vor allem im Norden Serbiens stark vertreten war, gehörte. Deswegen ist ihre Muttersprache auch das Ungarische und nicht das Serbokroatische, wie vielfach in der neuen Umgebung Schweiz vermutet wird. Die Familie befand sich sozusagen auch vor ihrer Ausreise in der Minderheit innerhalb einer anderssprachigen Mehrheit. Verstärkend kommt hinzu, dass die beiden Mädchen im Haus der Großmutter, dem „Prototyp eines Hauses, das die ersten und tiefsten Geheimnisse birgt“ (T. 12), auf eine ganz spezifische Welt zurückgeworfen sind, in der Mamika (wie die Großmutter von den Mädchen genannt wird) gerade in den ersten Lebensjahren zu einem Mutterersatz für die Erzählerin wird.

4 Erst 1973 - die Erzählerin ist fünfjährig - holen die Eltern ihre beiden Töchter in die Schweiz nach. Die Großmutter ist bis zu diesem Zeitpunkt stets die Brücke zu den 
Eltern in der Schweiz und beeinflusst auch die Wahrnehmung dieser dem Mädchen noch ganz unbekannten Welt:

„Svájcba, hatten Sie manchmal gesagt, Vater und Mutter seien in der Schweiz, in einer besseren Welt. Und wissen Sie, wie ich mir diese bessere Welt vorgestellt habe? ,Besser ' bedeutete für mich einfach ,mehr'. Mehr von allen guten Dingen, die ich kannte." (T. 173)

Da das Verhältnis mit der geliebten Großmutter in der Vojvodina ausgesprochen eng ist, wird der Wechsel in die Schweiz von Ildikó als sehr schwierig, sogar traumatisch empfunden. Zwar gibt es eine Art Übergangszeit, da die Großmutter die Mädchen auf ihrer Reise über Belgrad in die Schweiz begleitet und dort einige Zeit als Unterstützung bei ihnen bleibt. Als die Großmutter dann endgültig in ihre Heimat zurückkehrt, spürt das Kind den Verlust der vertrauten Lebenswirklichkeit und auch der ungarischen Muttersprache, in der sie immer mit der Großmutter kommuniziert hat. Das Ergebnis ist zunächst eine ausgeprägte Sprachlosigkeit im neuen Land und die Suche nach einer Identität, die sich aus mehrsprachigen und mehrkulturellen Facetten zusammensetzt.

Im Roman werden sowohl der Spracherwerb Ildikós von den Anfängen bis zur virtuosen Beherrschung der deutschen Sprache beschrieben und reflektiert als auch (damit zusammenhängende) Fragen der Integration in die Schweizer Gesellschaft berührt. Das Kind bewegt sich zwischen verschiedenen Sprachebenen: Ungarisch, Schweizerdeutsch und Hochdeutsch ${ }^{8}$. In der Familie selbst wird meist Ungarisch gesprochen. Auch nach vielen Jahren bewegen sich die Eltern noch nicht sicher in der deutschen Sprache (auch bleibt ein starker Akzent), wodurch Ildikó und ihre Schwester Nomi in die Rolle des Dolmetschers (z.B. bei Behörden) gebracht werden; auch helfen sie den Eltern bei den Vorbereitungen für den Staatsbürgerschaftstest, der Voraussetzung für die Einbürgerung in der Schweiz ist.

7 In der Schule sprechen die Kinder eher Schweizerdeutsch als Hochdeutsch. Auf Ildikó lässt sich das beziehen, was Melinda Nadj Abonji mehrfach in Artikeln und Interviews über ihre Schulerfahrungen berichtet hat. Sie beschreibt, wie eine Lehrerin versuchte, das sich noch nicht sicher in der neuen Sprache bewegende Kind zu „integrieren“, indem es ihm in einem Theaterstück die Rolle eines Tannenbaums zudachte. So gut gemeint diese Aufgabe gewesen sein mag, so sehr verstärkte sich doch das Gefühl von Stummheit und Sprachlosigkeit bei dem Kind'. Vermutlich hängt auch mit solchen Erfahrungen zusammen, dass der Spracherwerb bzw. das Lesenlernen bei der Autorin selbst mit einer Verzögerung von mindestens einem halben Jahr verlief ${ }^{10}$. Erst sehr spät - während eines Stipendienaufenthaltes an der Universität Graz - gelangt Melinda Nadj Abonji durch ein Initiationserlebnis ${ }^{11}$ schließlich zum literarischen Schreiben.

Ähnlich wie es die Autorin beschrieben hat, entdeckt Ildikó das Hochdeutsche als eigene, von Büchern geprägte, kreative und offene Welt. Im Gegensatz zum Schweizerdeutschen, in dem sie weitgehend sprachlos bleibt, und dem Ungarischen, das an die Welt der Eltern und der Herkunft geknüpft ist, kann sie im Hochdeutschen eine ihr auch in der Gegenwart Anerkennung einbringende Identität entwickeln.

9 In Tauben fliegen auf bewegt sich die Protagonistin permanent zwischen den verschiedenen Sprachebenen:

„[...]; ich war noch nicht lange in der Schweiz, und ich erinnere mich an viele schlaflose Nächte, und oft, wenn ich meine Eltern belauscht hatte, wirbelten die Wörter in meinem Kopf wie Laub an einem regnerischen, stürmischen Herbsttag, Wörter auf Ungarisch wie Papiere, Polizei, Briefe, dankbar, deutsche Wörter wie Familiennachzug, Schwarzarbeit, [...].“ (T. 48) 
In dieser Passage wird auch deutlich, wie sensibel Ildikó auf die verschiedensprachigen Wörter reagiert, die sich in ihrem Kopf überlagern, ohne sie in einen übergeordneten Kontext bringen oder in ihrer tieferen Bedeutung erfassen zu können. Durch die reale Schlaflosigkeit des Kindes und die Unruhe, die im Bild von Sturm und Regen aufgehoben ist, wird allerdings die emotionale Wirkung von Wörtern auf das Kind sichtbar. Dabei geht es immer um Wörter, die mit der Migration und deren Folgen zu tun haben. Nimmt man sie zusammen mit der im Roman an anderer Stelle erwähnten Schwarzenbach-Initiative ${ }^{12}$ und den darauf folgenden Volksentscheiden in den 70er Jahren, die leicht zu einer Abschiebung der Familie Kocsis hätten führen können, so wird die reale Bedrohung, der auch das Kind ausgesetzt ist, greifbar.

$11 \mathrm{Zu}$ betonen ist an dieser Stelle die Funktion der Protagonistin als sprachgrenzüberschreitende und -vermittelnde Figur. Zunehmend lernt das Mädchen mit der Sprache zu spielen, Assoziationen zu entwickeln. Auffällig ist, dass einzelne Wörter emotional an Bedeutung und Lebendigkeit gewinnen, vor allem weil sie bildhaft sind und die Vorstellungswelt des Kindes ansprechen, während andere Wörter kaum Kontur bekommen und daher schnell in den hinteren Regionen des Gehirns abgelegt werden:
„Irgendwann einmal habe ich das Wort ,Schwarzarbeit' aufgeschnappt, von meinen Eltern, ich konnte noch kaum Deutsch, und es gab Wörter, die vergass ich rasch wieder, andere gingen mir nicht mehr aus dem Kopf, so auch Schwarzarbeit. Es gefiel mir, dass ich das Wort zwar nicht brauchen konnte - im Gegensatz zu schlafen, essen, trinken, der See, die Frau, der Mann, das Kind, ja, nein - mir aber trotzdem vieles darunter vorstellen konnte. Andere Wörter hingegen stapelten sich in meinem Kopf wie nutzloser Kram: Ausweis, Niederlassung, Wartefrist. Bis ich die Bedeutung dieser Wörter begriff, dauerte es lange, auch deshalb, weil meine Eltern sie auf ihre Art betonten oder unabsichtlich abänderten. Der Ausweis war der Eisweis, die Wartefrist die Wortfrisch und Niederlassung klang aus ihrem Mund wie Niidärlasso." (T. 46f.)

\section{Zum Verhältnis von deutscher und ungarischer Sprache im Roman}

12 Im Roman von Melinda Nadj Abonji finden sich eine Reihe von Passagen, die direkt das Verhältnis der deutschen und der ungarischen Sprache beleuchten. Den beiden Sprachen werden bestimmte gesellschaftliche Bereiche zugeordnet, die sich zum Teil überlagern, zum Teil aber auch inkompatibel bzw. unüberbrückbar sind.

Die ungarische Sprache ist für Ildikó untrennbar mit ihrer Herkunft und der Großmutter in der Vojvodina verbunden ${ }^{13}$. Dabei gilt es $\mathrm{zu}$ betonen, dass das Ungarische in der Vojvodina weitgehend an den Kindheitskosmos im Haus der geliebten Großmutter (Mamika) geknüpft ist. Melinda Nadj Abonji macht das in einem Aufsatz anhand des Wortes „Haus“ klar. Während das ungarische Wort für Haus - „ház“ - „ein ebenerdiges Haus mit einem großen Korridor" ${ }^{\text {"14 }}$ und einem Innenhof bezeichnete, bedeutete "Haus" in der Schweiz etwas ganz anderes: Das schweizerdeutsche Haus war mehrstöckig, hatte einen Vorplatz, und in den Treppenhäusern waren rote Blumen mit Blättern, die sich pelzig anfühlten, auf Fenstersimsen aufgereiht; vor allem aber war das schweizerdeutsche Haus ausgehöhlt, und diese Höhle, in der die Autos standen, wurde ,Garage ' genannt. “" ${ }^{15}$ Dieses einfache Beispiel belegt sehr gut, welche Implikationen Wörter im Werk von Nadj Abonji haben. 
Während es sich bei dem Haus der Großmutter in der Vojvodina um ein ausgesprochen einfaches Einfamilienhaus mit Garten handelt, erlebt das Kind in der Schweiz fast nur Mehrfamilienhäuser mit unterirdischen Garagen. Doch über solche äußerlichen Unterschiede hinaus, geht es hier auch um den Bedeutungshof, der um Wörter herum gruppiert ist. So ist das ungarische Wort mit einem ganzen Kosmos einer geliebten, von "Stimmen" ${ }^{\text {"16 }}$ geprägten Kinderwelt im Haus der Großmutter in der Vojvodina verbunden, in dem auch ungarische Volkslieder und Redewendungen permanent präsent waren. Was das Kind mit Haus verbindet, ist eine sehr einfache, eher ländliche, aber emotional stark besetzte Kinderwelt. In der Schweiz hingegen wird Haus eher mit Anonymität, Ordnung und Perfektion verbunden.

Dass schon einzelne Wörter in beiden Sprachen so Unterschiedliches bezeichnen, führen bei Ildikó zu der Erkenntnis, dass „das Wesentliche [...] unübersetzbar“ (T. 310) ist.

Das Verhältnis der beiden Sprachen wird im Roman vor allem auch in Verbindung mit den Eltern Ildikós behandelt. Der Sprachgebrauch des Vaters beispielsweise, der weder Hochdeutsch noch das Schweizerdeutsch wirklich beherrscht, kann als typisch für die erste Einwanderergeneration bezeichnet werden, die mit keinen oder geringen Sprachkenntnissen in die Schweiz gekommen ist, sich die deutsche Sprache mehr oder weniger ungesteuert angeeignet hat und dementsprechend auch nach vielen Jahren noch fehlerhaft und mit starkem Akzent spricht. Bei ihm sind ganze Bereiche der Sprachverwendung ans Ungarische geknüpft, während er sich deutsche bzw. schweizerdeutsche Wörter oft nicht merken kann bzw. sie falsch ausspricht oder unverständlich betont. Sobald der Vater emotional berührt ist, wechselt er ins Ungarische ${ }^{17}$ : „Hülye csíny“ (T.100) [was etwa mit „dummer Kinderstreich“ zu übersetzen wäre], sagt der Vater zum Beispiel auf Ungarisch zur Tochter; und seine Flüche bringt er generell auf Ungarisch zum Ausdruck - wie Ildikó mutmaßt, „um zu verhindern, dass seine Muttersprache auskühlt, solange das Fluchen noch fliesst, können die geliebten Wörter doch unmöglich absterben, oder?“ (T.165) Gerade die emotionale Ebene der Flüche mag Ildikó an ihrem Vater, führt aber gleichzeitig auch zu Reflexionen über die Sprache und weckt den Wunsch, die Bildhaftigkeit der ungarischen Flüche in die deutsche Sprache zu übertragen:

„[...]und ich wünschte mir, ich könnte Vaters Flüche hörbar machen, so in die andere Sprache übersetzen, dass sie wirklich glänzen - Stalin, schickst du uns einen sibirischen Gruss aus deinem unterkühlten Arsch, willst du uns jetzt schon die Laune verderben, noch bevor wir angekommen sind, Adam und Eva, die immer noch unschuldig und besitzlos sind, nackt in deinem sozialistischen Paradies herumlaufen?

[...]

(Und wenn es möglich wäre, Vaters Wendungen in die andere Sprache, ins Deutsche $\mathrm{zu}$ überführen, dann könnte ich ihm zeigen, dass ich seine Art sich fluchend oder schweigend mitzuteilen, verstehe. Wenn nämlich bereits ein Wort keine Entsprechung findet, wie soll dann ein halbes Leben in der neuen Sprache erzählt werden?, dann kann nur das Schweigen oder die verkürzte, dramatische Form des Fluches davon erzählen, wie es gewesen ist, wie es gewesen sein könnte; das würde ich Vater sagen, und wahrscheinlich wäre er erstaunt darüber, dass ich mir über ihn, über seine Sprache Gedanken mache.)“ (T. 165)

16 Angedeutet wird in der Passage, dass eine Übersetzung ins Deutsche, die dem ungarischen Original gleichkommt, schon bei einzelnen Wörtern, aber umso mehr bei der Artikulation von Erfahrungen, nicht möglich ist. Damit benennt Ildikó ein von ihr 
empfundenes tieferreichendes Paradox, nämlich dass sie den Vater in seiner Art der sprachlichen Artikulation zwar versteht, aber nicht in der Lage ist, ihm das auch sprachlich mitzuteilen. Dadurch bleibt eine Kommunikationslosigkeit zwischen beiden, deren Ausdrucksform im Roman eben sehr häufig das Schweigen ist. Gegenüber der Außenwelt bleibt ebenfalls das Gefühl der Unmöglichkeit der Übertragung ganzer Erfahrungsbereiche, auch kultureller Besonderheiten, die in der Schweiz nicht verstanden werden.

\section{Sprache und Gedächtnis - Annäherung und Sprachlosigkeit}

Ist der Sprachwechsel in der ersten Generation der Migranten einer nicht vorhandenen umfassenden Sprachkompetenz im Deutschen geschuldet, so geht die zweite Generation der secondos in der Schweiz eher spielerisch mit den beiden Sprachen um. Auf die Frage eines Cousins, ob sie verliebt sei, antwortet Ildikó z.B. in einer Mischung beider Sprachen: „szerelmes, ja, bis über beide Ohren, sagt Nomi, szerelmet, füstöt, köhegést nem lehet eltitkolni, Liebe, Rauch und Husten könne man nicht verheimlichen, [....].“ (T. 203). Beide Sprachen stehen wie selbstverständlich in einem Satz nebeneinander, erhellen sich gegenseitig, zeigen aber auch, dass eine Übersetzung nicht so einfach funktioniert.

Redewendungen funktionieren ebenso wie Flüche oftmals nur in einer Sprache, und eine wörtliche Übersetzung kann daher meist bloß eine unbefriedigende Annäherung an den Ausgangsgehalt sein. Durch den Einbezug von Wörtern und ganzen Sätzen bzw. Redewendungen in den literarischen Text, dem fast immer eine deutsche Übertragung folgt, stellt Melinda Nadj Abonji beide Sprachen unkommentiert nebeneinander. Zum einen entsteht dadurch beim Lesen das Gefühl einer besonderen Bedeutung, aber auch Fremdheit und Hybridität, zum anderen aber werden Splitter einer anderssprachigen Vergangenheit der Protagonistin mit einbezogen. Und es wird sichtbar, dass trotz ausgezeichneter Sprachkenntnisse Ildikós und ihrer Freunde ein Bereich zwischen den Sprachen bleibt, der unübersetzbar ist. Bettina Spoerri verweist in ihrem Aufsatz " stifmutter und galtbir. Literarische Kreation auf der Schnittstelle von Sprachen "18 darauf, dass Melinda Nadj Abonji und Ilma Rakusa immer wieder in Veranstaltungen und Poetikvorlesungen betont haben, „dass sie mit ihrer zweiten, angelernten Sprache Deutsch nicht in die tiefen emotionalen Schichten ihrer Kindheit eindringen können, und das wirke sich auch deutlich auf ihre literarischen Texte aus. In der deutschen Sprache ist für sie beide der Zugang zu vorbewussten Bereichen verstellt, [...].“ ${ }^{19}$ Die erste Sprache werde von Nadj Abonji als „Speicher von Erinnerungen und sinnlichen Eindrücken “ ${ }^{{ }^{20}}$ verstanden, die in der neuen Sprache fehlen. Insofern kann ihre Literatur auch gelesen werden als Suche nach einem kulturellen Gedächtnis der Herkunftskultur, deren Übertragung in die deutsche Sprache allerdings vielfach versperrt ist. Dieser für den Roman von Melinda Nadj Abonji zentrale Aspekt der Rolle der Sprache für die Repräsentation eines bikulturellen Gedächtnisses wird immer wieder von Ildikó reflektiert. Ihre Sprachlosigkeit in vielen Situationen resultiert daraus, dass „bereits ein Wort keine Entsprechung findet" und es ihr daher fast unmöglich erscheint, ,ein halbes Leben in der neuen Sprache“ (T. 165) zu erzählen. Vor allem betrifft das einen großen, mit Stimmen, Empfindungen, Farben und Tönen verbundenen Komplex, die „Atmosphäre meiner Kindheit“ (T. 19) : „[...] aber wie sagt 
man, dass man eine Ebene liebt, die Pappeln, staubig, gleichgültig, stolz, und die Luft dazwischen?“ (T. 8)

In den ersten Jahren nach dem Landwechsel fährt die Familie Kocsis regelmäßig zurück in die Vojvodina, um die Großmutter zu besuchen. Ildikó hat schon als Kind große Angst vor jeder Veränderung der Welt der Großmutter, die sie daher konservieren möchte, um nicht den Boden unter den Füßen zu verlieren: „Das Erkennen der immergleichen Gegenstände, die mich vor der Angst schützt, als Fremde in dieser Welt dazustehen, von Mamikas Leben ausgeschlossen zu sein, [...]“ (T. 13). Als die Großmutter dann im Wendejahr 1989 stirbt, bedeutet das einen großen Einschnitt im Leben Ildikós, die nach der Beerdigung zehn Jahre lang nicht mehr in ihre Heimat fährt. Von nun an muss die Erinnerung an die Welt in der Vojvodina aus dem Gedächtnis hervorgeholt bzw. konstruiert werden.

\section{Formen der Polyphonie}

20 Wurden im vorausgegangenen Abschnitt die in mancherlei Hinsicht auch unüberwindbaren sprachlichen Grenzen betont, so findet sich im Roman auch eine Ebene der sprachlichen Polyphonie, die als eine Art Utopie vorgestellt wird. Während Ildikó große Probleme hat, Elemente ihrer Herkunft in die neue Sprache zu übertragen und sich damit ganze Bereiche dem deutschsprachigen Erzählen in der Schweizer Umgebung widersetzen, so entsteht mit dem serbischen Kriegsflüchtling Dalibor aus Dubrovnik eine von mehreren Sprachen geprägte Liebesbeziehung, die es Ildikó ermöglicht, bislang in ihren Alltag nicht integrierbare Teile ihrer Vergangenheit mit einzubeziehen. Mit Dalibor, der nur wenig Deutsch spricht, unterhält sie sich meist auf Englisch, aber auch auf Serbokroatisch und Ungarisch (und ansatzweise auf Deutsch) über Themen, über die sie sich sonst mit niemandem vorher in der Schweiz unterhalten hat, „ein zerfleddertes Wörterbuch zwischen uns, das uns verbindet, [...]“ (T. 184), so über die Vojvodina und die Lage ihrer Verwandten dort während des Balkankrieges. Zentral für die Beziehung mit Dalibor ist, dass verschiedene Sprachen integrierbar sind und der Grad an Perfektion in ihrer Beherrschung nicht als der ausschlaggebende Faktor für das Verständnis zwischen Menschen angesehen wird. Bestimmend ist eher die Bereitschaft, sich auf die verschiedenen Sprachebenen einzulassen und sich von ihnen berühren zu lassen, wodurch der Wunsch nach Verstehen wächst:

„[...] sein Sprechgesang, der der Frühlingsluft, dem dunklen See etwas in seiner Sprache erzählt (alle Lieder aller Sprachen müsste man doch verstehen, denke ich, Gott, der seine babylonische Sprachverwirrung auf die gesprochene Sprache hätte beschränken müssen; Dalibors Lied ist so schön und absichtslos gesungen, berührt mich so sehr, dass ich es unerträglich finde, seine Worte nicht zu verstehen).“ (T. 197)

21 Die Liebesbeziehung mit Dalibor kann als (allerdings nur kurzzeitiger) Gegenentwurf zu all den isolierten und schwer miteinander verbindbaren Sprachebenen im Roman angesehen werden. Darin gibt es bei allen Unterschieden - z.B. auch keiner wirklich gemeinsamen Sprache mit Dalibor - das Bemühen miteinander zu kommunizieren und sich gegenseitig zu verstehen. In der Passage wird allerdings ein ähnliches Paradox wie mit dem Vater sichtbar, nämlich dass der emotionalen Berühung (hier durch das Lied Dalibors) der Schmerz über den versperrten Zugang zur Bedeutung der einzelnen Wörter gegenüber steht. Sprache wird in dieser Passage weit über die Worte hinaus in 
seiner Wirkung auf Menschen gesehen. In der Liebesbeziehung mit Dalibor ist eine Vereinigung über die Grenzen der einzelnen Sprachen hinweg zumindest kurzzeitig möglich:

„[...] und er streckt meine Hände nach mir aus, wir umarmen uns, flüstern uns einzelne Worte ins Ohr, wir küssen uns, zum ersten Mal, es ist schön, dass du hier bist, wir küssen uns mehrsprachig, ich habe mich in dich verliebt, auf Ungarisch, Deutsch, Serbokroatisch, Englisch.“ (T. 198)

\section{Der Kreis schließt sich}

Der Roman Tauben fliegen auf von Melinda Nadj Abonji behandelt anhand Ildikós Weg auf sehr differenzierte Weise das Thema Spracherwerb, das zugleich die Suche nach einer stabilen Identität beinhaltet. Dabei spielt der Weggang aus der Vojvodina in die Schweiz insofern eine wichtige Rolle, als er mit dem Verlust alter Gewissheiten und Sprachlosigkeit verbunden ist. Die Suche nach einer mehrkulturellen und mehrsprachigen Identität, in der die verschiedenen Sprachen und Kulturen aufgehoben sind, ist zentrales Thema des Romans. Ildikó Kocsis bewegt sich zwischen verschiedenen Sprachen bzw. Sprachebenen, ist vor allem konfrontiert mit dem Schweizerdeutschen, dem Hochdeutschen und dem Ungarischen, darüber hinaus auch mit dem Serbokroatischen, dem Englischen und Formen der Schweizer Jugendsprache. Auffällig ist, dass sie mit allen diesen Sprachebenen bestimmte Realitätsbereiche verbindet, wie zum Beispiel mit dem Ungarischen die frühkindliche Welt im Haus der Großmutter bzw. die der Eltern. Emotional sind diese Bereiche stark belegt, wie anhand des Wortes „Haus/ház“ gezeigt werden konnte. Ildikó tritt zwar im Roman immer wieder als grenzüberschreitende und vermittelnde Figur auf, es bleibt aber die Erkenntnis, dass „das Wesentliche [...] unübersetzbar“ ist. Diese Erkenntnis impliziert sowohl Sprachlosigkeit wie ein hohes Maß an Isolation. In Tauben fliegen auf wird auch thematisiert, dass frühkindliche Erfahrungen Ildikós, die in einer anderskulturellen und anderssprachigen Umgebung gemacht wurden, sich einer Erinnerung in der deutschen Sprache versperren. Am ehesten gelingt eine Überwindung der Sprachgrenzen in der Liebesbeziehung mit dem Kriegsflüchtling Dalibor. Aufgehoben sind Sprachen auch in hybriden Passagen, in denen zum Beispiel das Ungarische unkommentiert neben das Deutsche gesetzt wird. Nicht zuletzt gelingt Melinda Nadj Abonji auch in der Titelmetapher eine Überwindung der Sprachgrenzen. Denn das Motiv der Tauben entstammt einem Lied, dass die geliebte Großmutter Ildikó in der Kindheit vorsang: „Von meiner Mutter habe ich das Herz einer Taube“ (T. 152). Gleichzeitig verweist es auf die bewegte Situation Ildikós in der Schweiz: „und ich sah uns, übergross, ich, eine aufgeregt flatternde Taube, von menschlichen Schritten aufgescheucht." (T. 145) 


\section{NOTES}

1. Der Hauptpreis ist mit $25000 €$ dotiert, die (in der Regel sechs) KandidatInnen auf der Shortlist erhalten jeweils $2500 €$. Die Verleihung, die immer am Vorabend der Frankfurter Buchmesse vorgenommen wird, hat zudem nachweislich eine hohe Auflage zur Folge.

2. Hingewiesen werden soll in diesem Kontext kurz auf den Adelbert-von-Chamisso-Preis der Robert Bosch Stiftung, der in München für AutorInnen nicht deutscher Muttersprache oder Herkunft vergeben wird.

3. Mit dem Schweizer Buchpreis wurde bereits im Jahr 2009 eine Autorin mit Migrationshintergrund, nämlich Ilma Rakusa, ausgezeichnet.

4. Die Begriffsbildung ist allerdings in Fachkreisen nicht unumstritten; häufig ist auch die Rede von interkultureller, transkultureller oder hybrider Literatur o.ä. Vgl. etwa: Volker C. Dörr: „Deutschsprachige Migrantenliteratur. Von Gastarbeitern zu Kanakstas, von der Interkulturalität zur Hybridität“, in: Karin Hoff (Hrsg.): Literatur der Migration - Migration der Literatur. Texte und Untersuchungen zur Germanistik und Skandinavistik, Frankfurt/M. 2008, Peter Lang, S. 17-33.

5. Der Begriff „Secondo/ Seconda“, der häufig in der Schweiz verwendet wird, meint ursprünglich italienische und spanische Gastarbeiter in den 60er und 70er Jahren und wird nun auf die zweite Generation der Migranten bezogen. Gemeint sind alle in der Schweiz geborenen Menschen mit ausländischen Eltern (oder auch solche, die aus Mischehen stammen) und Menschen, die in jungen Jahren in die Schweiz eingewandert sind. Vgl. dazu insbesondere: Martina Kamm, Bettina Spoerri, Daniel Rothenbühler, Gianni D’Amato: Diskurse in die Weite. Kosmopolitische Räume in den Literaturen der Schweiz, Zürich, Seismo Verlag, 2010.

6. Bei Zitaten aus diesem Roman wird in Klammern das Kürzel „T“ und die Seitenzahl angefügt.

7. Vgl. zu den sprachlichen Besonderheiten auch: Margrit Verena Zinggeler: How second generation immigrant writers have transformed swiss and german language literature: a study of authors from the Swiss ,secondo-space', Lewiston, New York, 2011, S. 149-151, 192-195.

8. Außerdem ist Ildikó konfrontiert mit dem Serbokroatisch der Serviererinnen aus dem ehemaligen Jugoslawien in der Cafeteria Mondial. Nach Ausbruch des Krieges auf dem Balkan wird der Gebrauch der Sprachen zunehmend konflikthaft. Im Szenetreff Wohlgroth in Zürich schließlich sprechen die Besucher einen spezifischen Jugendslang, der von englischen Wörtern und coolen Sprüchen durchsetzt ist. Zudem spricht sie mit Dalibor oft Englisch.

9. Vgl. Melinda Nadj Abonji: „Zu Hause in der Fremde - Versuche zur Integration“, in: Sprache im technischen Zeitalter 2011, Heft 198, S. 181-190, hier S. 182.

10. Ebd., S. 184.

11. Das Initiationsereignis ist die Offenheit eines kleinen Jungen im Schwimmbad, der sie ohne Erwartungen zum Tauchen einlud, vgl. ebd., S. 187f., und Margrit Verena Zinggeler: How second generation immigrant writers have transformed swiss and german language literature, a.a.O., S. 150.

12. James Schwarzenbachs Volksbegehren 1970 gegen die „Überfremdung“ in der Schweiz wurde knapp abgelehnt. Auch 1974 wurde ein Begehren gegen die Überfremdung in der Schweiz abgelehnt.

13. Sprache im technischen Zeitalter 2011, Heft 198, a.a.O., S. 183.

14. Ebd.

15. Ebd., S. 184.

16. „Meine Großmutter hatte keine Sprache, sondern eine Stimme.“, vgl. Willi Wottreng, „Melinda Nadj Abonji“, in: Ursula Binggeli: Mutter, wo übernachtet die Sprache? 14 Porträts mehrsprachiger Autorinnen und Autoren in der Schweiz, Zürich, Limmat Verlag, 2010, S. 106.

17. Auch die Mutter wechselt häufig in Gesprächen ins Ungarische bzw. streut ungarische Wörter in ihre Sätze ein, wenn ihr die deutsche Entsprechung nicht einfällt. Generell spricht sie mit 
anderen Migranten aus der Vojvodina Ungarisch, sofern diese nicht gut genug Deutsch können. Nicht selten fungiert dabei Ildikó als Übersetzerin bzw. „Wörterbuch“ für schwierige Ausdrücke. 18. Bettina Spoerri: „,stifmutter ${ }^{\star}$ und ,galtbir‘. Literarische Kreation auf der Schnittstelle von Sprachen“, in: Martina Kamm, Bettina Spoerri, Daniel Rothenbühler, Gianni D’Amato: Diskurse in die Weite, a.a.O., S. 126-139.

19. Ebd., S. 126.

20. Ebd., S. 127.

\section{RÉSUMÉS}

Melinda Nadj Abonji - deutschsprachige, in der Schweiz lebende Autorin ungarisch-serbischer Herkunft - reflektiert in ihrem preisgekrönten Roman Tauben fliegen auf (2010) auf vielschichtige und interessante Weise das Thema Sprache. Der Aufsatz geht v.a. anhand der Figur der Erzählerin Ildikó Kocsis, Tochter einer Einwandererfamilie aus der Vojvodina (Serbien), der Frage nach, welche Schwierigkeiten beim Erwerb der deutschen Sprache (bzw. des Schweizerdeutschen) und dem Versuch einer Integration in der Schweiz entstehen. Fokussiert wird auch auf das Verhältnis von ungarischer Muttersprache und deutscher Sprache, die in Verbindung damit thematisierten Grenzen der Kommunikation (bis hin zur Sprachlosigkeit) und einer bikulturellen Erinnerung sowie die Suche nach einer mehrkulturellen bzw. mehrsprachigen Identität. Ildikó stößt immer wieder an Grenzen der Artikulation, wenn es darum geht, Elemente aus ihrer Vergangenheit in der Vojvodina in ihre deutschsprachige Umgebung zu integrieren. Daher können Passagen im Roman, in denen zwei Sprachen unkommentiert nebeneinander stehen oder in Beziehung zueinander treten, als Versuch verstanden werden, monolinguale Begrenzungen zu überwinden.

Melinda Nadj Abonji - a German-language author of Hungarian-Serbian origin, who has lived in Switzerland since childhood - engages in a multilayered and interesting way with the meaning of language in her prize-winning novel Tauben fliegen auf (2010). Speaking through the lens of the first-person narrator, Ildikó Kocsis, the daughter of immigrant parents from the Vojvodina region in Serbia, this article explores the difficulties migrants face in terms of acquiring the Swiss-German language and integrating into Swiss society. Moreover, It highlights how the narrator's Hungarian mother tongue impacts on German and how communication barriers emerge from the linguistic limitations of each language. In addition, It analyses how Ildikó's memory and personality were shaped by two cultures, which in turn resulted in a multicultural and multilingual identity. Time and again, Ildikó is faced with linguistic limitations when she tries to incorporate her previous life experiences in the Vojvodina into a German-speaking environment. Thus, we can interpret those sections in Nadj Abonji's novel in which two languages are placed next to each other or where two languages are related to one another as a means to transcend monolingual linguistic and conceptual barriers.

Melinda Nadj Abonji - auteur de langue allemande, d'origine serbo-hongroise résidant en Suisse - propose dans son roman Tauben fliegen auf (2010/Pigeon vole) - le roman a reçu le Prix allemand du livre - une réflexion à plusieurs niveaux et fort intéressante sur le thème de la langue. À partir du personnage de la narratrice Ildikó Kocsis, fille d'une famille d'immigrés de la Voïvodine (Serbie), l'article s'interroge sur les difficultés qu'il y a à faire sienne la langue allemande (en 
l'espèce, le suisse allemand) et à tenter de s'intégrer en Suisse. L'éclairage porte aussi sur les relations entre la langue maternelle hongroise et la langue allemande et, lié à cette question, sur les limites de la communication (jusqu'au silence) en présence d'une mémoire biculturelle, il porte encore sur la quête d'une identité pluriculturelle, voire plurilingue. Ildikó se heurte constamment aux limites de l'articulation possible lorsqu'il y va d'intégrer dans son environnement germanophone des éléments de son passé dans la Voïvodine. Ceci permet de voir dans les passages du roman où deux langues sont juxtaposées sans le moindre commentaire ou bien sont mises en relation l'une avec l'autre, une tentative pour dépasser les limites auxquelles condamne le monolinguisme.

\section{AUTEURS}

\section{RENÉ KEGELMANN}

Universität München 